\title{
DYNAMICS OF CORONAL LOOP OSCILLATIONS RECENT IMPROVEMENTS AND COMPUTATIONAL ASPECTS
}

\author{
T. VAN DOORSSELAERE*, I. ARREGUI, J. ANDRIES, M. GOOSSENS \\ and S. POEDTS \\ Centrum voor Plasma-astrofysica, Celestijnenlaan 200B, B-3001 Leuven, Belgium \\ (*Author for correspondence: E-mail: tomvd@wis.kuleuven.be)
}

(Received 29 July 2005; Accepted in final form 9 September 2005)

\begin{abstract}
We will discuss the observed, heavily damped transversal oscillations of coronal loops. These oscillations are often modeled as transversal kink oscillations in a cylinder. Several features are added to the classical cylindrical model. In our models we include loop curvature, longitudinal density stratification, and highly inhomogeneous radial density profiles.

In this paper, we will first give an overview of recently obtained results, both analytically and numerically. After that, we shed a light on the computational aspects of the modeling process. In particular, we will focus on the parallellization of the numerical codes.
\end{abstract}

Keywords: MHD, plasmas, Sun: corona, Sun: oscillations

\section{Introduction}

Coronal loop oscillations were first observed with TRACE by Schrijver et al. (1999), Aschwanden et al. (1999), and Nakariakov et al. (1999). A more extensive study was performed by Schrijver et al. (2002) and Aschwanden et al. (2002), who published the observational properties of 11 such events. Recently, Verwichte et al. (2004) detected both fundamental and overtone oscillations in a coronal loop arcade. While all these oscillations are perpendicular to the coronal loop plane, vertical oscillations have recently been reported by Wang and Solanki (2004). It is generally accepted that the nature of these oscillations is a fast magnetosonic kink $(m=1)$ oscillation. Often they are interpreted in terms of quasi-modes (see e.g., Ruderman and Roberts, 2002). These quasi-modes are damped by resonant absorption, which actually rapidly converts the fast kink mode to torsional Alfvén waves. The generated Alfvén waves are then available to heat the coronal loop (Moriyasu et al., 2004) to observed temperature profiles (Landi and Landini, 2004).

Analytical calculations in cylindrical configurations of the frequency and damping times of such quasi-modes were published by Hollweg and Yang (1988), Goossens et al. (1992), and Goossens et al. (1995). In these analytical derivations, it is assumed that the radial inhomogeneity is quasi-discontinuous. Numerical calculations concerning more realistical radial inhomogeneity profiles were done by Hollweg (1990) and Van Doorsselaere et al. (2004a).

Space Science Reviews (2005) 121: 79-89

DOI: $10.1007 / \mathrm{s} 11214-006-2963-5$

(C) Springer 2006 
The observed oscillations also provide an excellent tool for coronal seismology. Nakariakov and Ofman (2001) used them to obtain an estimate for the value of the magnetic field in coronal loops and Goossens et al. (2002) and Aschwanden et al. (2003) exploited these oscillations to calculate the density ratio (internal/external) for a set of loops and compared it with the observed values. They found an agreement between the observed and calculated values within a factor 2 , which is excellent, given the observational constraints.

\section{Classical Model and Improvements}

An often used model applies linearized MHD to a static equilibrium and neglects gravitation and gas pressure (plasma- $\beta=0$ ). Additionally, the curvature of the loop is neglected and cylindrical geometry is assumed. The equilibrium magnetic field is taken in the $z$-direction only and is assumed to be uniform everywhere.

$$
\vec{B}=B \vec{e}_{z} .
$$

Consequently, the equilibrium density profile can be chosen freely. The coronal loop is then modeled by a density enhancement. Often a model with two cavities of constant density is used, the internal loop and the external medium. These two cavities are then connected at the loop radius $R$ with a layer of thickness $l$ (see Figure 1), where the density smoothly varies from the internal to the external value.

The perturbations are Fourier-analyzed in the $z$ - and $\theta$-direction (with Fourier numbers $k_{z}$ and $m$ ).

Obvious improvements of the model are to include curvature, to allow variation of the density along the loop, to add nonlinear effects, to include chromospheric upflows and footpoint leakage, ...

The first two are discussed in Sections 3 and 4.

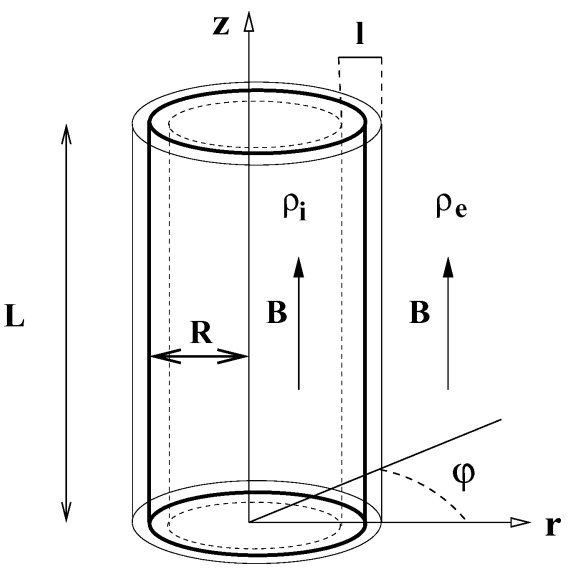

Figure 1. Sketch of the classical cylindrical model. 


\section{Curvature}

Because we want to curve our initially straight cylindrical coronal loop, we have to employ another coordinate system. Following Van Doorsselaere et al. (2004b), we use toroidal coordinates. They are described by the transformation formulae:

$$
x=\frac{a \sinh u \cos \phi}{\cosh u-\cos v}, \quad y=\frac{a \sinh u \sin \phi}{\cosh u-\cos v}, \quad z=\frac{a \sin v}{\cosh u-\cos v},
$$

where $(x, y, z)$ are the original Cartesian coordinates, $(u, v, \phi)$ are the new toroidal coordinates, and $a$ the (large) radius of the torus. As can be seen from Figure 2, $u$ is the new (dimensionless) radial coordinate, $v$ the poloidal coordinate, and $\phi$ the toroidal coordinate, equivalent with $z$ in the cylindrical model.

When taking a constant $u$-surface, a torus is outlined, thus making a good model for a curved coronal loop.

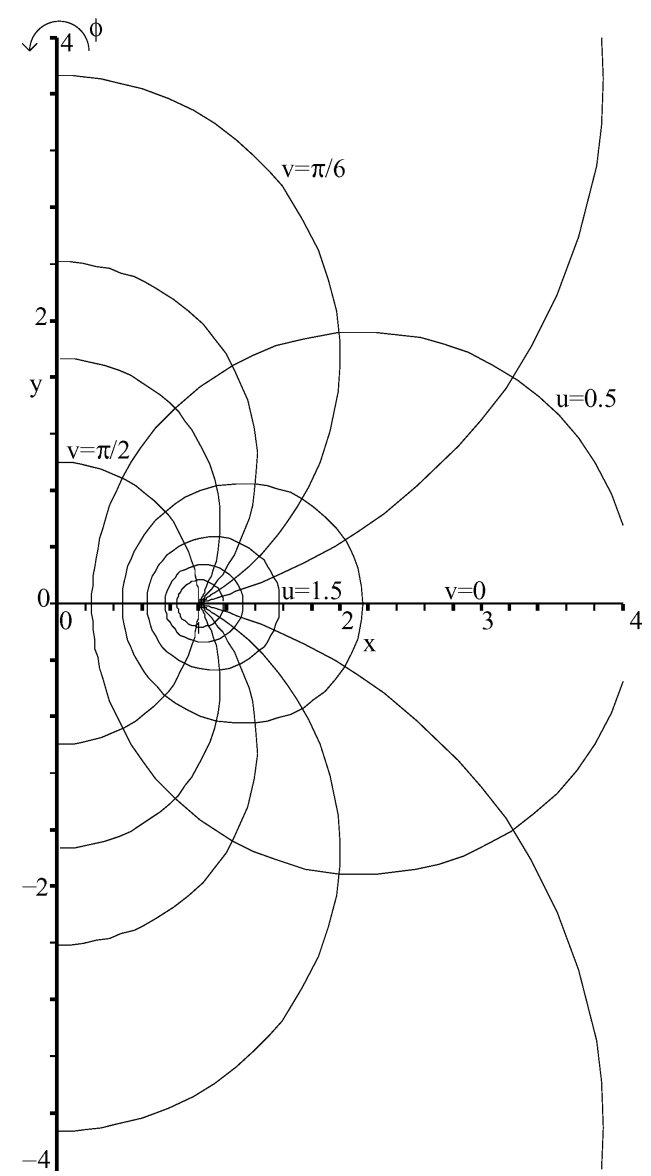

Figure 2. The toroidal coordinate system for $a=1$. 
Because, in this geometry, a uniform magnetic field is not force-free, a better equilibrium magnetic field has to be found. Solving the current-free condition together with the solenoidal constraint for the magnetic field yields a unique form

$$
\vec{B}=B(u, v) \vec{e}_{\phi}=B_{\mathrm{a}} \frac{(\cosh u-\cos v)}{\sinh u} \vec{e}_{\phi} .
$$

Here $B_{\mathrm{a}}$ is the magnetic field at $u \rightarrow \infty$, i.e., the axis of the torus.

The governing equations for the linear perturbations are

$$
\begin{aligned}
\rho \frac{\partial \vec{V}}{\partial t} & =\frac{1}{\mu}(\vec{\nabla} \times \vec{b}) \times \vec{B}, \\
\frac{\partial \vec{b}}{\partial t} & =\vec{\nabla} \times(\vec{V} \times \vec{B}),
\end{aligned}
$$

where $\vec{b}=\left(b_{u}, b_{v}, b_{\phi}\right)$ and $\vec{V}=\left(V_{u}, V_{v}, V_{\phi}\right)$ are the perturbations of the magnetic field and the velocity, respectively. Using the toroidal coordinate system and Fourier-analyzing in the $\phi$-direction (wavenumber $k$ ), these can be expanded to

$$
\begin{aligned}
\frac{\partial^{2} \vec{V}}{\partial t^{2}}+\omega_{\mathrm{A}}^{2} \vec{V}= & V_{\mathrm{A}}^{2} \vec{\nabla}_{\perp}\left(\frac{1}{B} \frac{\partial b_{\phi}}{\partial t}\right), \\
\frac{\partial b_{\phi}}{\partial t}= & -\frac{B}{a} \sinh u(\cosh u-\cos v) \\
& \times\left\{\frac{\partial}{\partial u}\left(\frac{V_{u}}{\sinh u}\right)+\frac{\partial}{\partial v}\left(\frac{V_{v}}{\sinh u}\right)\right\} .
\end{aligned}
$$

Here we defined the Alfvén speed by $V_{\mathrm{A}}^{2}=B^{2} / \mu \rho$ and the Alfvén frequency as $\omega_{\mathrm{A}}^{2}=\frac{V_{A}^{2} k^{2}(\cosh u-\cos v)^{2}}{a^{2} \sinh ^{2} u}$.

Now the density may be chosen so that two cavities with constant Alfvén frequency are obtained:

$$
\rho(u, v)= \begin{cases}\rho_{\mathrm{a}, \mathrm{e}} \frac{(\cosh u-\cos v)^{4}}{\sinh ^{4} u} & \text { for } u<u_{0}, \\ \rho_{\mathrm{a}}(u) \frac{(\cosh u-\cos v)^{4}}{\sinh ^{4} u} & \text { for } u_{0} \leq u \leq u_{0}+d, \\ \rho_{\mathrm{a}, \mathrm{i}} \frac{(\cosh u-\cos v)^{4}}{\sinh ^{4} u} & \text { for } u>u_{0}+d .\end{cases}
$$

$u_{0}$ is the outer boundary of the coronal loop, and $u_{0}+d$ the inner boundary. $\rho_{\mathrm{a}, \mathrm{i}}$ and $\rho_{\mathrm{a}, \mathrm{e}}$ are the densities at the axis of the coronal loop, respectively for the inner part, (subscript i) and the outer corona (subscript e). $\rho_{\mathrm{a}}(u)$ is arbitrary function continuously connecting the internal and external region. 
Using this density profile, Equations (1)-(3) can be reduced to a single equation for $b_{\phi}$ in both the internal and external region:

$$
\nabla^{2}\left(\frac{b_{\phi}}{B}\right)=-\left(\frac{\kappa^{2} \omega^{2}(\cosh u-\cos v)^{2}}{\omega_{A, \mathrm{i} / \mathrm{e}}^{2} a^{2} \sinh ^{2} u}\right) \frac{b_{\phi}}{B} .
$$

The general solution to this equation can be written as

$$
\sum_{m=-\infty}^{+\infty} C_{\mathrm{i} / \mathrm{e}, \mathrm{m}} \sqrt{\frac{\cosh u-\cos v}{\sinh u}} F_{\mathrm{i} / \mathrm{e}, \mathrm{m}}(u) \exp \left(i m\left(v-v_{0}\right)\right) .
$$

In this solution, $C_{\mathrm{i} / \mathrm{e}, \mathrm{m}}$ are constant coefficients, $F_{\mathrm{i} / \mathrm{e}, \mathrm{m}}(u)$ gives the radial dependency (which can be expressed in terms of hypergeometric functions, see Van Doorsselaere et al., 2004b) and $v_{0}$ is an arbitrary phase. This phase is determined by the form and direction of the incoming shock wave, which triggers the coronal loop oscillation. Thus, every phase can be attained, and vertical as well as horizontal oscillations are allowed.

It is also clear from Equation (4) that different poloidal mode numbers $m$ are now coupled (through the $v$-dependency under the square root).

By using the appropriate connection formulae (see e.g., Sakurai et al., 1991) between the internal and external region, a dispersion relation may be found. Expanding this dispersion relation in the small curvature limit $(\varepsilon=R / a \ll 1)$, shows that the frequency is modified to:

$$
\omega=\omega_{0 \mathrm{r}}-i \omega_{0 \mathrm{i}}\left(1+\varepsilon \frac{\frac{3}{4}}{m^{2}+\frac{1}{2}}\right),
$$

where

$$
\begin{aligned}
& \omega_{0 \mathrm{r}}=\frac{2 \omega_{\mathrm{Ae}}^{2} \omega_{\mathrm{Ai}}^{2}}{\omega_{\mathrm{Ae}}^{2}+\omega_{\mathrm{Ai}}^{2}}, \\
& \omega_{0 \mathrm{i}}=-\frac{\frac{\pi \omega_{0 \mathrm{r}}^{2}}{|\Delta|}\left(\frac{\omega_{0 \mathrm{r}}^{2}}{\omega_{\mathrm{Ai}}^{2}}-1\right)\left(\frac{\omega_{0 \mathrm{r}}^{2}}{\omega_{\mathrm{Ae}}^{2}}-1\right)\left(m^{2}+\frac{1}{2}\right)}{2|m| \omega_{0 \mathrm{r}}\left(\frac{\omega_{\mathrm{Ae}}^{2}+\omega_{\mathrm{Ai}}^{2}}{\omega_{\mathrm{Ae}}^{2} \omega_{\mathrm{Ai}}^{2}}\right)},
\end{aligned}
$$

which are actually the real and imaginary part of the oscillation frequency in the cylindrical case. Interesting to note is that only the imaginary part of the frequency is influenced by the curvature. The damping of the coronal loop oscillation is only slightly stronger in a curved configuration than in a straight flux tube. Since the influence of the curvature on the frequency is so small (only $12 \%$ more damping), it is unlikely that this effect can be observed with the current instruments. Additionally, it can be observed that the frequencies do not depend on $v_{0}$. We can thus conclude that a vertical and horizontal oscillation must have the same frequency and damping rate in the limit of small curvature. 
The connection formulae can also be expanded in $\varepsilon$ and show that, in the small curvature limit, a quasi-mode oscillation with poloidal mode number $m$ only couples to neighboring poloidal mode numbers $m \pm 1$.

\section{Density Stratification}

In this section, we return to the cylindrical model of Section 2. Instead of adding curvature, a density stratification along the loop is assumed. We now assume a density profile of the form:

$$
\rho(r, z)=\rho(r)\left(1-\alpha \sin \left(\frac{\pi}{L} z\right)\right) .
$$

$0 \leq \alpha<1$ models the stratification in the $z$-direction. For $\alpha=0$, we have an unstratified coronal loop equivalent to the model in Section 2. For $\alpha=1$, we have an unphysical coronal loop with an empty loop top.

Analytical results can be obtained for $\alpha \ll 1$ (see Andries et al., 2005b). Assuming that the frequency has only a first order (in $\alpha$ ) correction, it can be shown that

$$
\omega=\omega_{0}+\alpha \omega_{1}=\omega_{0}-\frac{\alpha}{2} \omega_{0} S_{k, k}=\omega_{0}\left(1-\frac{\alpha}{\pi}\left[1-\frac{1}{1-4 k^{2}}\right]\right) .
$$

Here $k$ is the longitudinal mode number. Thus, it can be observed that the change $\alpha \omega_{1}$ to the frequency in the unstratified medium $\omega_{0}$ is proportional to $\omega_{0}$ itself. Therefore, we can conclude that the ratio of the real part and the imaginary part of the frequency is unchanged by the longitudinal density stratification.

More generally, for higher $\alpha$ but small $l / R$, the eigenfrequencies and eigenfunctions can be calculated by connecting the analytical solutions in the two homogeneous layers with a numerically integrated solution in the connecting layer. The pressure perturbation and radial displacement are shown for the fundamental mode in Figure 3. Clearly, coupling to higher order mode numbers is present in the pressure perturbation. Since the density perturbation is proportional to the pressure perturbation, additional maxima should be detected in the light curve along the coronal loop while it is oscillating.

When more realistical coronal loop models, i.e., with smoother density profiles, are considered, a numerical code has to be used. Arregui et al. (2005) used POLLUX, a two-dimensional numerical code with finite elements in the radial direction and a spectral discretization in the $z$-direction. The code is an eigenvalue code and was introduced by Van der Linden (1991). The code uses the JacobiDavidson shooting method to find eigenvalues close to a given target eigenvalue.

A large parameter scan is performed and the dependency on $\alpha$ is investigated. The resulting plots can be seen in Figure 4. These figures exhibit the dependence 

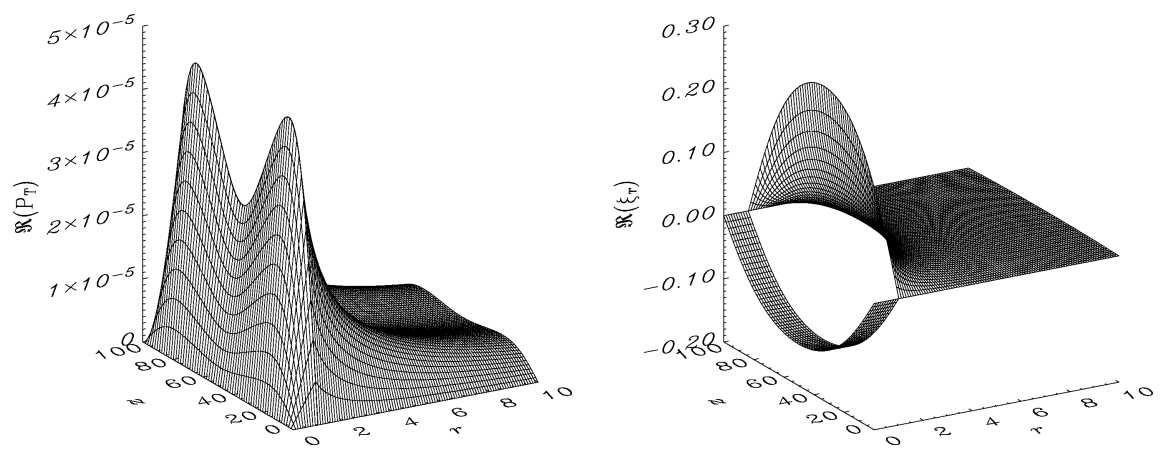

Figure 3. Real part of the pressure perturbation and radial displacement. Figures for the fundamental kink mode and for $\alpha=0.78$.
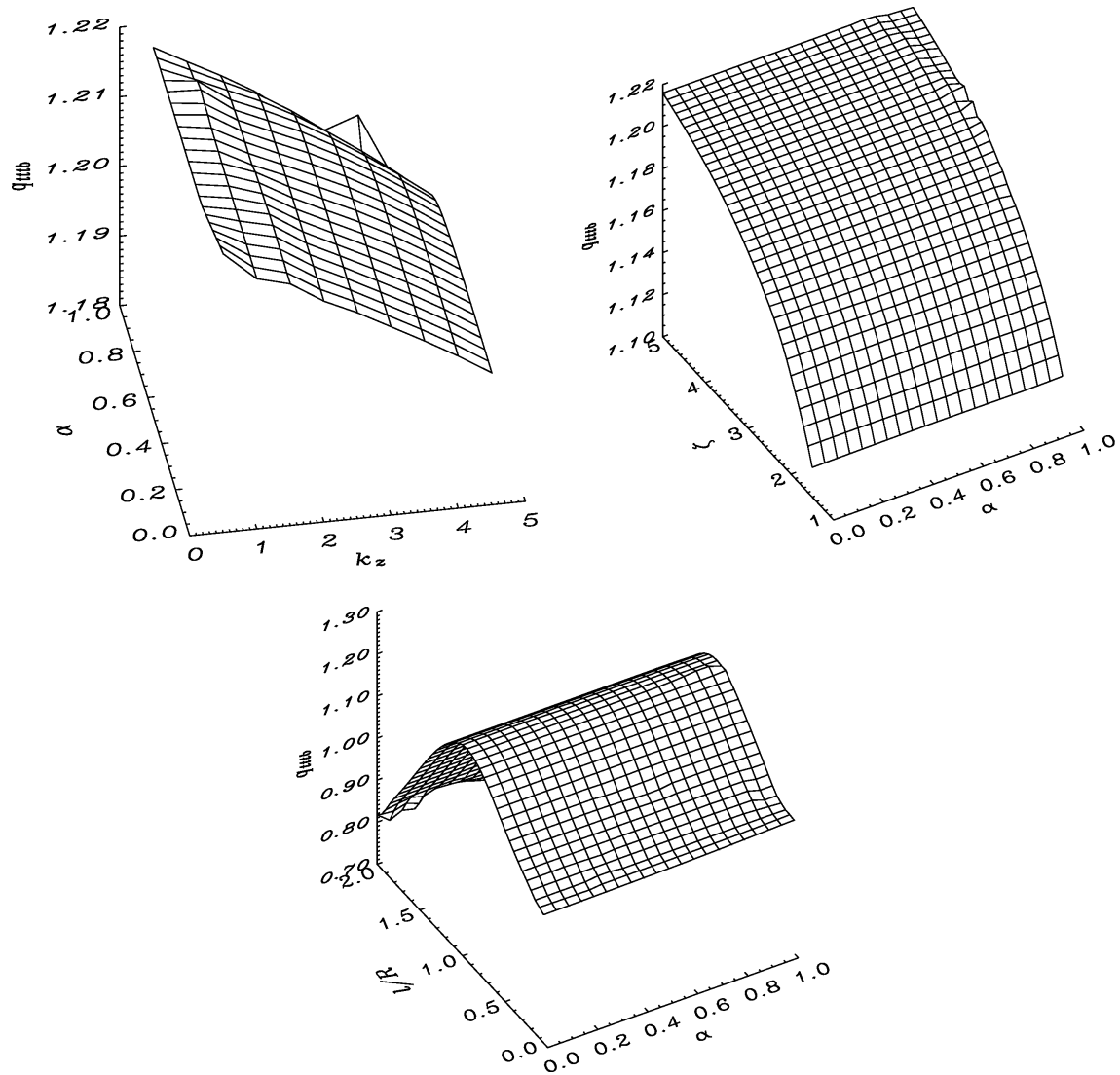

Figure 4. Top left: $q_{\mathrm{TTTB}}$ vs. $k_{z}$ and $\alpha$ for $l / R=1$ and $\zeta=5$; top right: $q_{\mathrm{TTTB}}$ vs. $\zeta$ and $\alpha$ for $l / R=1$ and $k_{z}=0.04$; Bottom: $q_{\mathrm{TTTB}}$ vs. $l / R$ and $\alpha$ for $\zeta=4$ and $k_{z}=0.08$. 


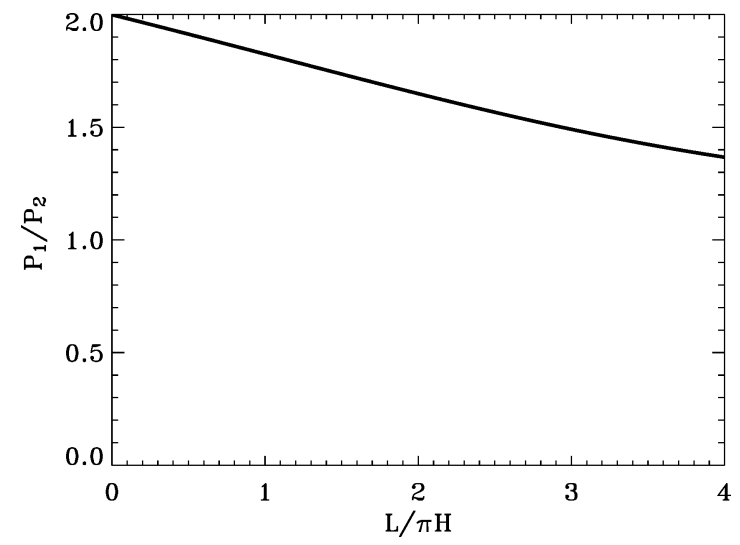

Figure 5. The ratio of the periods of the fundamental mode and the first overtone vs. the density scale height $H$.

of $q_{\text {Tттв }}$ on the longitudinal wavenumber $k_{z}$, the density contrast $\zeta$, and the width of the inhomogeneous layer $l / R . q_{\text {Тттв }}$ is defined by

$$
-\frac{\omega_{\mathrm{i}}}{\omega_{\mathrm{r}}}=-q_{\mathrm{TTTB}} \frac{1}{4} \frac{l}{R} \frac{\zeta-1}{\zeta+1},
$$

i.e., it is $\omega_{\mathrm{i}} / \omega_{\mathrm{r}}$ normalized to the analytical value obtained with the model in Section 2. $q_{\text {Tттв }}=1$ means that the analytical formula is perfectly true.

From these figures it is clear that the analytical prediction for small stratification remains true for larger values of $\alpha$ and thick inhomogeneous layers. The only variation in these figures is the same as found by Van Doorsselaere et al. (2004a).

The theory of longitudinally stratified coronal loops is excellent to do coronal loop seismology. Because of the stratification, the ratio of the periods of the fundamental kink mode $P_{1}$ and the first overtone $P_{2}$ will significantly differ from 2 . When calculating this ratio for different density scale heights $H$, a one to one relation is obtained (see Figure 5). Consequently, when two periods are measured in the same coronal loop, the density scale height in this loop can be inferred.

Andries et al. (2005a) used the values found by Verwichte et al. (2004) to find a density scale height in two coronal loops. Their results can be found in Table I. In

TABLE I

Density scale heights inferred from the observed ratio of the periods of the fundamental kink mode and the first overtone.

\begin{tabular}{lll}
\hline$P_{1} / P_{2}$ (from Verwichte et al., 2004) & $H($ in Mm) & Confidence interval (in Mm) \\
\hline $1.81 \pm 0.25$ & 65 & ]$-\infty,-190] \cup[27,+\infty[$ \\
$1.64 \pm 0.23$ & 36 & {$[20,99]$} \\
\hline
\end{tabular}


two cases they find a scale height of respectively 65 and $36 \mathrm{Mm}$, which is compatible with the observational value of $50 \mathrm{Mm}$.

\section{Computational Aspects}

Normally, parameter studies are perfect for parallellization, because no interaction between two cases is needed. Often a linear speedup is shown in these programs. Our case, however, is different from a standard parameter study, because a target is needed for the shooting method. Since the target changes for each parameter set, a massively parallel algorithm is not possible.

We assume that we have a two-dimensional parameter space (e.g., a scan over $\zeta$ and $l / R$ ) and that every case takes exactly the same CPU time. We then assign a master node to scan over the $\zeta$-dimension for the smallest $l / R$ value. The master node's results can then be transmitted to the compute nodes, who iterate over the $l / R$ dimension and take the transmitted value as an initial target. By using this method, only one target has to be provided to the program. The consequent targets can be taken as the results from the previous steps (if the step sizes are small enough).

A closed formula for the needed computation time for $N$ processors can be found. If there are $a$ steps in the $l / R$-direction and $b$ steps in the $\zeta$-direction,

$$
((b-1) \operatorname{div} N) a+(b-1) \bmod N+a,
$$

steps are needed to complete the computation. $x$ div $y$ and $x \bmod y$ return respectively the quotient and remainder of $x$ divided by $y$. Multiplying this formula with the time needed for one iteration yields the total time needed to compute the problem. A comparison between this theoretical value and actual timings can be found in Figure 6.

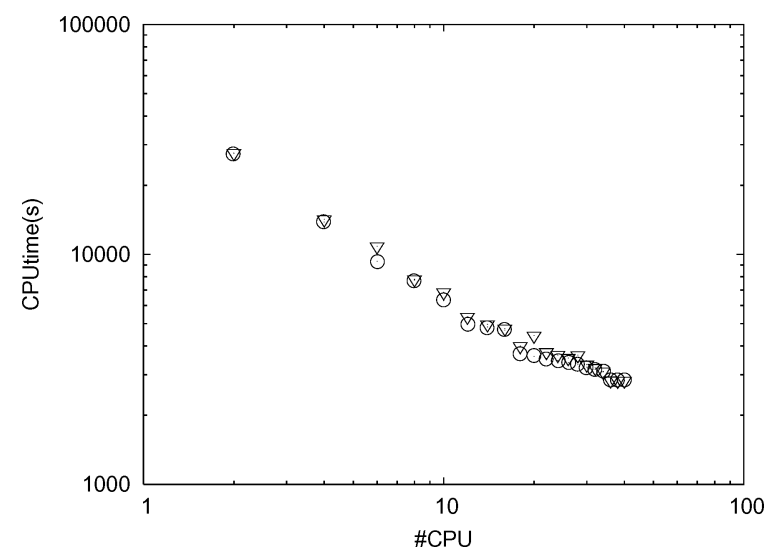

Figure 6. POLLUX code, $\nabla$ indicates the actual timings, $\odot$ indicates the theoretically predicted values. Values obtained for 36 and 41 steps in respectively the $\zeta$ - and $l / R$-dimension. 
More generally, if three dimensions are to be scanned over, the total number of steps can be computed by

$$
((c b-1) \operatorname{div} N)) a+(c b-1) \bmod N+a,
$$

where $c$ is the number of iterations in the third dimension.

The used parameter space in Section 4 scanned over a $9 \times 21 \times 36 \times 41$-grid. On a single CPU such a computation would need $10.4 \mathrm{Ms} \approx 120$ days to complete. Using a cluster of 64 CPUs, this computation theoretically only needs $181 \mathrm{ks} \approx 2$ day $3 \mathrm{~h}$.

\section{Conclusions}

We can conclude that we significantly improved the classical cylindrical model for a coronal loop. Curvature and longitudinal density stratification were added. As a result of the curvature, coupling between different poloidal mode numbers occurs and quasi-mode oscillations are slightly (up to 12\%) more damped. Additionally, it is shown that a poloidal phase may be chosen freely. Thus, curvature does not select preferential oscillation directions: Vertical as well as horizontal oscillations are still possible, as observed recently.

Density stratification along the coronal loop, on the other hand, couples longitudinal mode numbers. The change of the frequency is proportional to the frequency itself. Consequently, the ratio of the imaginary and the real part of the frequency does not change.

Our model for longitudinally stratified coronal loops has proven to be a valuable tool for coronal loop seismology. Andries et al. (2005a) inferred values of 65 and $36 \mathrm{Mm}$ for the density scale height in two cases where the fundamental mode and an overtone were observed in the same loop. These values for the density scale height is compatible with the observed value of $50 \mathrm{Mm}$.

We parallellized our numerical code and found a closed formula which describes the speedup. Because the code is not massively parallel, for a large number of processors the speedup decreases. In practice, however, for a realistical amount of CPUs good speedup factors are obtained.

\section{Acknowledgements}

For his precious comments and help while parallellizing the POLLUX-code, the authors would like to thank Dries Kimpe. These results were obtained in the framework of the projects GOA 2004/01 and OT 02/57 (K.U.Leuven), G.0451.05 (FWOVlaanderen), and 90203 (ESA Prodex 8). 


\section{References}

Andries, J., Arregui, I., and Goossens, M.: 2005a, ApJ 624, L57.

Andries, J., Goossens, M., Hollweg, J. V., Arregui, I., and Van Doorsselaere, T.: 2005b, A\&A 430, 1109.

Arregui, I., Van Doorsselaere, T., Andries, J., Goossens, M., and Kimpe, D.: 2005, A\&A 441, 361.

Aschwanden, M. J., De Pontieu, B., Schrijver, C. J., and Title, A. M.: 2002, Solar Phys. 206, 99.

Aschwanden, M. J., Fletcher, L., Schrijver, C. J., and Alexander, D.: 1999, ApJ 520, 880.

Aschwanden, M. J., Nightingale, R. W., Andries, J., Goossens, M., and Van Doorsselaere, T.: 2003, ApJ 598, 1375.

Goossens, M., Andries, J., and Aschwanden, M. J.: 2002, A\&A 394, L39.

Goossens, M., Hollweg, J. V., and Sakurai, T.: 1992, Solar Phys. 138, 233.

Goossens, M., Ruderman, M. S., and Hollweg, J. V.: 1995, Solar Phys. 157, 75.

Hollweg, J. V.: 1990, J. Geophys. Res. 95, 2319.

Hollweg, J. V., and Yang, G.: 1988, J. Geophys. Res. 93, 5423.

Landi, E., and Landini, M.: 2004, ApJ 608, 1133.

Moriyasu, S., Kudoh, T., Yokoyama, T., and Shibata, K.: 2004, ApJ 601, L107.

Nakariakov, V. M., and Ofman, L.: 2001, A\&A 372, L53.

Nakariakov, V. M., Ofman, L., DeLuca, E. E., Roberts, B., and Davila, J. M.: 1999, Science 285, 862.

Ruderman, M. S., and Roberts, B.: 2002, ApJ 577, 475.

Sakurai, T., Goossens, M., and Hollweg, J. V.: 1991, Solar Phys. 133, 227.

Schrijver, C. J., Aschwanden, M. J., and Title, A. M.: 2002, Solar Phys. 206, 69.

Schrijver, C. J., Title, A. M., Berger, T. E., Fletcher, L., Hurlburt, N. E., Nightingale, R. W., et al.: 1999, Solar Phys. 187, 261.

Van der Linden, R. A. M.: 1991, PhD Thesis, K.U. Leuven.

Van Doorsselaere, T., Andries, J., Poedts, S., and Goossens, M.: 2004a, ApJ 606, 1223.

Van Doorsselaere, T., Debosscher, A., Andries, J., and Poedts, S.: 2004b, A\&A 424, 1065.

Verwichte, E., Nakariakov, V. M., Ofman, L., and Deluca, E. E.: 2004, Solar Phys. 223, 77.

Wang, T. J., and Solanki, S. K.: 2004, A\&A 421, L33. 